\title{
The Nano Gold Rush: Graphynes as Atomic Sieves for Coinage and Pt-Group Transition Metals
}

\author{
Sunkyung Kim ${ }^{\mathrm{a}, \mathrm{b}}$, Pablo Gamallo ${ }^{\mathrm{c}}$, Francesc Viñes ${ }^{\mathrm{c}, *}$, Jin Yong Lee ${ }^{\mathrm{d}, * *}$ \\ a Department of Chemistry, Sungkyunkwan University, Suwon 16419, Korea \\ ${ }^{\mathrm{b}}$ Department of Basic Science, Korea Air Force Academy, Cheongju 28187, Korea \\ ${ }^{\mathrm{c}}$ Departament de Ciència de Materials i Química Física \& Institut de Química Teòrica i \\ Computacional (IQTCUB), Universitat de Barcelona, c/Martí i Franquès 1, 08028
}

Barcelona, Spain

\begin{abstract}
Graphyne two-dimensional materials have been envisaged as potential useful membranes for applications such as gases separation and water desalination with very high salt rejection rates. The graphyne acetylenic linkages length defines its acetylenic holes size, thus offering a way of tuning their membrane permeability. The present study evaluates, based on density functional theory simulations, the possible use of $\gamma$-graphyne, the graphyne with the smallest acetylenic hole, to sieve Transition Metal (TM) atoms. The systematic study comprises obtaining adsorption minima for the $303 d, 4 d$, and $5 d$ TMs along with transition states for the diffusion across the $\gamma$-graphyne layer. The study reveals very small penetration barriers, below $0.5 \mathrm{eV}$, for late $3 d$, Pt-group, and coinage metals, whereas water molecules are found to display high penetration energy barriers above $5 \mathrm{eV}$, even when accounting for possible $\gamma$ graphyne out-of-plane deformations facilitating the water trespassing. The adsorption energy distribution of the adsorbed TMs shows that $\mathrm{Pd}$ and $\mathrm{Au}$ sieving by $\gamma$-graphyne would be specially enhanced versus their anchoring on the $\gamma$-graphyne carbon framework, thus pointing $\gamma$-graphyne as a possible effective membrane to sieve particularly late transition metals.

*\&** Corresponding authors: francesc.vines@ub.edu, jinylee@,skku.edu
\end{abstract}




\section{Introduction}

In the latest years graphynes, two-dimensional (2D) carbon allotropes containing $\mathrm{C}$ atoms both with $s p$ and $s p^{2}$ hybridization states, have become a hub of research [1-3]. Theoretically hypothesized by Balaban et al. as early as in 1968 [4], their study remained latent for decades, with the sole exception of Baughman and coworkers one in the eighties [5], until theoretical studies on them by Density Functional Theory $(D F T)$ predicted the existence of Dirac cones in their band structure [6]. This introduced graphynes as competitors to graphene on charge carrier transport, with conductivities similar or even better than those of graphene [7]. These forecasted properties boosted the posterior experimental research on graphynes, with significant advances in the graphyne synthesis [8-10] and the experimental corroboration of the anticipated DFT results [11].

Such encouraging results have fostered further research on graphynes for practical applications. These include the usage of graphdiyne as support for $\mathrm{TiO}_{2} \mathrm{P} 25$ photocatalyst $[9,12]$ improving its activity towards methylene blue photodegradation by an efficient charge transportation and a good coupling of graphdiyne electronic structure with that of $\mathrm{TiO}_{2}$. Furthermore, graphynes have been posed as convenient matrices for Li-based batteries [13], templates on single-atom anchoring [14] catalysts for the Oxygen Reduction Reaction (ORR) [15], and even, Ca-decorated, adequate materials for $\mathrm{H}_{2}$ storage $[16,17]$.

A particular appealing and currently rising field of application of $\gamma$-graphynes is using them as membranes, given that they naturally display pores in their Carbon framework; see the example of $\gamma$-graphyne in Fig. 1. The different acetylenic connectivity and the lengths of the chained $s p$ bonds define the graphyne phase, e.g. $\alpha-, \beta$-, $\gamma$-graphyne, etc. [6], as well as the size and shape of the featured acetylenic pores. The research on the use of graphynes as membranes accompanies other studies on different carbon allotropes; for instance nanoporous graphene has been proposed as a membrane for water desalination via reverse osmosis [18], 
or Carbon Nanotubes $(C N T s)$ are contemplated as water channels $[19,20]$. Even graphene oxide frameworks have been studied for water desalination purposes [21], thus placing carbon based materials in general under the spotlight of membrane research [22].

As far as graphynes are concerned, graphdiynes - graphynes with links consisting of four $s p \mathrm{C}$ atoms connecting an hexagonal close-packed array of benzene rings - have been computationally studied for the separation or isolation of gas molecules. For instance, Cranford and Buehler have studied, by means of the reactive Force Field (ReaxFF) potential, the trespassing of $\mathrm{H}_{2}, \mathrm{CO}$, or $\mathrm{CH}_{4}$ through graphdiyne, finding the selective diffusion of $\mathrm{H}_{2}$ with an estimated permeation energy barrier of solely $0.11 \pm 0.03 \mathrm{eV}$ [23]. In the same spirit, Bartolomei et al. have studied the penetration of $\mathrm{He}$ versus $\mathrm{CH}_{4}$ on graphdiyne by coupled supramolecular second order Møller-Plesset perturbation theory $(M P 2 C)$, finding an essentially unimpeded trespassing of $\mathrm{He}$, indicating an exceptionally high $\mathrm{He} / \mathrm{CH}_{4}$ separation over a wide range of temperatures [24], and even the possibility of separating ${ }^{3} \mathrm{He}$ and ${ }^{4} \mathrm{He}$ isotopes at low temperatures [25].

More fruitful has been the study of graphynes on water desalination. Diverse working groups reported, by Molecular Dynamics $(M D)$ simulations employing a variety of Force Fields $(F F s)$ and conditions, the same picture; graphtriyne, also known as graphyne-3, as well as graphyne-4, feature both a permeability to water - of the order of $40 \mathrm{~L} \mathrm{~cm}^{-2} \mathrm{~h}^{-1}$ - several times higher than the conventional reverse of forward osmosis membranes [26], even an order of magnitude higher than nanoporous graphene [27], with an essentially perfect $100 \%$ salt rejection [28]. Along this line, the rejection of nearly all ions in seawater seem to be achievable, including as well organic or inorganic contaminants [29,30], implying suitable trespassing energy barriers for $\mathrm{H}_{2} \mathrm{O}$ yet not for ions and molecules. Further than that, water flux through the walls of graphyne- 3 nanotubes is estimated to be 5 orders of magnitude 
higher than commercial forward osmosis membranes, while these membranes serve as salt ions traps [31].

Other MP2 calculations have shown that while $\mathrm{H}_{2} \mathrm{O}$ penetration across graphyne-3 layer is barrierless, it features energy barriers of $\sim 0.23$ and $\sim 8 \mathrm{eV}$ for graphdiyne and graphyne, respectively. However, the graphdiyne barrier is found to reduce to $0.09 \mathrm{eV}$ when mediated by a hydrogen bond to another water molecule situated at the opposite site of the graphdiyne pore [32]. This effect has been also observed by FF MD simulations on graphyne3, where the diffusion barrier for the trespassing of a single water molecule across was estimated to be of $0.06 \mathrm{eV}$ at $300 \mathrm{~K}$, somewhat smaller than CNT membrane barriers of 0.09 $\mathrm{eV}$ as obtained at the same conditions [33].

Clearly, the membrane capabilities of graphynes for small molecules and atoms such as $\mathrm{He}, \mathrm{H}_{2}$, and $\mathrm{H}_{2} \mathrm{O}$ are of great interest in many fields, as the above-mentioned gases (isotopes) separation, as well as water desalination or waste water cleaning purposes, evaluating which species can easily trespass the graphyne sheets, which cannot yet get strongly attached (trapped), and which essentially do not interact. A recent first-principles study based on Density Functional Theory (DFT) highlighted the enhanced trapping capability of $\gamma$-graphyne towards most of Transition Metal (TM) atoms [34] compared to a more densely packed carbon structure such as graphene [35], revealing the enhanced adsorption energies of $\gamma$-graphyne towards the TM adatoms, although the interaction for some TMs was still low, specially true for those TM with $d^{10}$ electronic configuration.

Given the aforementioned salt rejection capabilities of graphynes [31], mostly evaluated for alkaline or alkaline-earth halides, the $\mathrm{H}_{2} \mathrm{O}$ permeation possibilities [32], and the trapping possibilities of $\gamma$-graphyne for TMs adatoms [34], the remaining question mark here would be whether such a graphyne material would actually act as a trap for TM atoms or could even act as a sieve for them? To answer this question, we present here a thorough DFT 
study addressed at estimating the energy barriers associated to the diffusion of all $3 d, 4 d$, and $5 d$ TM atoms across $\gamma$-graphyne, chosen as a representative graphyne with the smallest pore size possible, thus contemplating the worst scenario for adatom trespassing across it.

Such a systematic study departs from the assumption of having TM adatoms on $\gamma$ graphyne, regardless of its origin, that can be multiple; from atomic deposition [36], to atomic detachment from adsorbed clusters or nanoparticles, e.g. carried out by soft landing [37] or grown on $\gamma$-graphyne, but considering as well TM adatoms coming from solvated TM cations in solution, either being adsorbed or reduced. Notice that different conditions are assumed or processes to have occurred depending on the origin; for instance, the atomic deposition at low coverage, the eventual loss of an atom of a deposited cluster or nanoparticle, or, in the case of solvated TM cations, their adsorption on $\gamma$-graphyne, reduced at a given applied bias or loosing part of its solvation energy, this latter point assuming the electronic reorganization of the adsorbed TM with graphyne, as deeply studied previously, where most of the adsorbed TM adatoms keep a certain degree of positive charge [34]. All this casuistry involves situations with mixed TMs, either being cationic components in solution, or deposited being part of a bimetallic deposited cluster or nanoparticle, yet, as aforementioned, one considers here low coverage situations of such TM adatoms, so as to consider that their coexistence does not alter their possible $\gamma$-graphyne trespassing, yet one has to keep in mind that the adsorption and trespassing energies may vary for larger coverages, specially when the TM adatoms can laterally interact among each other, even compete for available adsorption and penetration channel sites.

Notice that systematic earlier reports highlight that the adsorption strength of such independent TM adatoms on $\gamma$-graphyne is sensibly large [34], in some cases, larger than the parent TM bulk [14], and likely larger than such atoms in low-coordinated sites of clusters and nanoparticles; an aspect that supports the significant presence of atomically dispersed 
atoms. Likewise, generally, the TM adsorption strengths surpass the solvation energies of TM cations in solution [38], specially when considering that the adsorbed TM cation would still be partially hydrated, as the electronic rearrangement with the $\gamma$-graphyne substrate normally yields to positively charged species [34]. For late TMs with $d^{10}$ electronic configuration with small to negligible adsorption energies, there would be no energetic preference to partially desolvate and attach to $\gamma$-graphyne [39], yet could be forced, i.e. by cation reduction using a proper bias and profiting from the conducting properties of graphynes [6].

\section{Computational Details}

The spin polarized DFT calculations have been performed using the Vienna $A b$ initio Simulation Package —VASP [40]. The Projector Augmented Wave (PAW) method has been used to represent the atomic core electron density effect on the valence electron density [41]. This simulation of the core states allows one to obtain converged results —energy variations below $0.001 \mathrm{eV}$ - with a cut-off kinetic energy of $415 \mathrm{eV}$ for the plane-wave basis set. Geometry optimizations were performed using a conjugated gradient algorithm and applying a first-order Methfessel-Paxton smearing of $0.2 \mathrm{eV}$ width, yet final energies were corrected to $0 \mathrm{~K}$ (no smearing). The convergence threshold for the total energy was set to $10^{-5} \mathrm{eV}$ and structural optimizations were finalized when forces acting on atoms were below $0.01 \mathrm{eV} \AA^{-1}$. All DFT calculations have been carried out using the PBE exchange-correlation $(x c)$ functional [42], a representative xc functional within the Generalized Gradient Approximation $(G G A)$. PBE values provide an optimized pristine $\gamma$-graphyne rhombohedral lattice structure with unit cell parameters of $6.880 \AA$ (i.e., $a=b$ ), in very good agreement with previous estimates $[43,44]$ and has been proven to yield the best overall description of TMs bulks and surface properties among many Local Density Approximation ( $L D A)$, GGA, meta-GGA, and hybrid xc functionals [45-47]. 
Energy and structure optimizations have been carried out on a $p(2 \times 2)$ slab supercell, since it grants a separation between adsorbed adatoms of $\sim 1.3 \mathrm{~nm}$, enough to avoid interactions with TM adatoms on periodically repeated adjacent cells, and so is adequate to model low coverage situations. Test calculations on the supercell effect showed variations of adsorption energies below $0.02 \mathrm{eV}$ and structural changes below 3\% [34], as well variations on Bader charges below 0.02 e going towards smaller coverages, yet can start to be nonnegligible for larger coverages, from $0.12 \mathrm{eV}$ upwards, as studied earlier [14]. A vacuum region of $1 \mathrm{~nm}$ is added along the direction normal to the $\gamma$-graphyne layer, in order to avoid interactions between repeated slabs. Test calculations with double vacuum yielded variations in the energy of $\sim 0.003 \mathrm{eV}$. An optimal Monkhorst-Pack $\Gamma$-centred k-point grid of $2 \times 2 \times 1$ dimensions was used [48]. The van der Waals (vdW) dispersive interaction has not been considered, as previous studies, which included vdW via e.g. D2 dispersion correction of Grimme [49], showed that the vdW contribution of TM interaction to graphene and $\gamma$ graphyne is almost anecdotic, as most of the interaction is electronic in nature $[34,35]$. The adsorption energy values, $E_{a d s}$, of TM adsorbed on $\gamma$-graphyne are taken from literature, and obtained as

$$
E_{a d s}=\left(E_{G Y}+E_{T M}\right)-E_{T M / G Y}
$$

where $\mathrm{E}_{\mathrm{TM} / \mathrm{GY}}$ is the total energy of $\gamma$-graphyne layer with the TM adatom attached, $\mathrm{E}_{\mathrm{GY}}$ is the total energy of the pristine $\gamma$-graphyne layer, and $\mathrm{E}_{\mathrm{TM}}$ the total energy of an isolated $\mathrm{TM}$ atom in vacuum, as previously calculated [45]. Notice that we deliberately use the isolated TM in vacuum in its ground electronic state as a reference for all the cases, as is the one most clearly defined and reproducible, even though one has to keep in mind the possible origin of the TM adatom in a practical situation, which can be diverse, e.g. as above stated, coming from solvated cations, detached from adsorbed clusters, etc., with the different casuistry of the 
origin, including the number of solvating water molecules, or the crystallographic phase of the supported cluster, as well as its size and shape.

For any TM atom adsorbed on $\gamma$-graphyne the energy barrier for the diffusion across the $\gamma$-graphyne layer, $E_{\text {diff, }}$ is obtained as the difference in energy of TM in-plane in the middle of the acetylenic ring, hereafter denoted as $\mathbf{H}_{3}$ position, see Fig. 1, and the most stable adsorption site, as reported before [34], being normally the position right above the acetylenic ring, and here considered and denoted $\mathbf{H}_{1}$. Notice that such definition comes from the characterization of $\mathbf{H}_{3}$ as a Transition State (TS), see below. Note that these results are taken from a systematic study of the TM adsorptive landscape over $\gamma$-graphyne, carried out in a likewise fashion, and exploring other hollow sites, such as over the benzene ring, as well as different non-equivalent bridge and top sites. For more details, we refer to the literature [34]. There are few TMs whose most stable position is already $\mathbf{H}_{3}$, in particular, $\mathrm{Co}, \mathrm{Ni}, \mathrm{Cu}$, and $\mathrm{Re}$, and its characterization already provided in an earlier study [34]. In such cases, the $E_{\text {diff }}$ has been acquired finding the transition state along the removal path of the TM atom from $\mathbf{H}_{3}$ and its placement on $\mathbf{H}_{\mathbf{1}}$. In all cases, the diffusion energy barrier, $E_{\text {diff, }}$, is gained as

$$
E_{\text {diff }}=E_{T S}-E_{T M / G Y}
$$

where $E_{T S}$ is the energy of the located TS, and $E_{T M / G Y}$ is the energy of the of the TM adsorbed on most stable site. As above stated, that normally implies $\mathbf{H}_{\mathbf{1}}$, and $\mathbf{H}_{\mathbf{3}}$ position being the TS.

In almost all the cases an $\mathbf{H}_{\mathbf{3}}$ state have been found and characterized as a TS by vibrational frequency analysis, see below. Only on few cases where no direct $\mathbf{H}_{\mathbf{3}}$ was gained by optimization, or those where it was found to be a minimum, vide supra, the energy profile for the diffusion across the layer has been acquired by TS location obtained in a point-wise fashion, where the adatom height position with respect to $\gamma$-graphyne is kept fixed, as well as a $\gamma$-graphyne remote $\mathrm{C}$ atom - origin $x$ and $y$ coordinates, whose movement is frozen along the three-dimensional space, whereas all other $\mathrm{C}$ atoms and degrees of freedom are allowed to 
relax. In this way, the drifting of the full $\gamma$-graphyne to recover the TM adatom minimum is avoided, yet the $\gamma$-graphyne layer is allowed to deform to better accommodate the TM at a given height. Minima and TS have been characterized by all positive and only one imaginary vibrational frequency, respectively, obtained from the diagonalization of the Hessian matrix by finite differences of analytical gradients with individual displacements of $3 \mathrm{pm}$ in each cell direction.

\section{Results and Discussion}

As above commented, after optimization of $\mathbf{H}_{\mathbf{3}}$ and $\mathbf{H}_{\mathbf{1}}$ positions, they were characterized as either minima or TSs by a vibrational frequency analysis, finding none or one imaginary frequency, respectively. In the cases where no TS was characterized, it was found via pointwise optimizations, and the saddle point duly characterized by a sole imaginary frequency. The estimated energy barriers for the diffusion of TMs across the $\gamma$-graphyne layer, $E_{\text {diff, }}$ were then gained and listed in Table 1, whereas their $d$ series trends are captured in Fig. 2. Note that, generally speaking, the diffusions are prohibitive, typically $E_{\text {diff }}$ values being well above $1 \mathrm{eV}$, although clear trends are observed along the $d$ series, in the sense that when one goes along a $d$ series, the atomic radii reduce. Consequently, the atoms gradually fit better in the $\gamma$ graphyne hole, and, because of that, display reduced $E_{\text {diff }}$ values, showing a decay along the series. Exceptions to this trend are $d^{10} \mathrm{TMs} \mathrm{Zn}, \mathrm{Cd}$, and $\mathrm{Hg}$, that, by having their $d$-shell full occupied, display significantly larger radii, and so, larger $E_{\text {diff. Finally, those TMs that }}$ displayed adsorption minima at $\mathbf{H}_{3}, \mathrm{Re}, \mathrm{Co}, \mathrm{Ni}$, and $\mathrm{Cu}$, exhibit very high diffusion barriers, highlighting their large in-plane stability [34]. A significant electronic influence in the course of the diffusion is discarded as a Bader charges analysis in between $\mathbf{H}_{\mathbf{1}}$ and $\mathbf{H}_{\mathbf{3}}$ provides, in mean terms, differences below $0.1 e$.

A more important finding is that the $\mathrm{Cr}, \mathrm{Mn}$, and Fe $3 d$ metals, and specially the scarce and expensive coinage and Pt-group metals Rh, Pd, Ag, Ir, Pt, and Au metals, present 
appealing and affordable $E_{\text {diff }}$ values below $0.5 \mathrm{eV}$. Specifically, Fe, Pd, Pt, and Au feature extremely low energy barriers of solely $0.04,0.02,0.02$, and $0.01 \mathrm{eV}$, respectively, which strongly point for an extremely rapid diffusion across the $\gamma$-graphyne membrane. These results already point the use of $\gamma$-graphyne for sieving such TMs instead of just serving as a chemical trap, although here still the adsorption energy aspect has to be taken into account, vide infra. A further analysis of this $\gamma$-graphyne rapid diffusion can be gained by considering whether the energy released by the adsorption of these TMs on $\mathbf{H}_{\mathbf{1}}$ is enough to overcome $E_{\text {diff. }}$ In order to inspect this, we firstly calculated to what fraction of the adsorption energy, $E_{a d s}$, corresponds $E_{\text {diff. }}$ This quantity, given in percentage, Q, is plotted along $d$ series in Fig. 3. A previous study highlighted that the diffusion energy of diverse species over TM surfaces is a constant fraction of the adsorption energy [50], and by that, implying a rugosity of the potential energy landscape dictated by the interaction of the adatom or adsorbed molecule and the substrate surface. However, the results in Fig. 3 directly reveal that this is not the case for trespassing energy barriers, displaying a variety of Q values, and so, if any, one can recognize different behaviours, e.g. discriminating early from late TMs, and $3 d$ late TMs behaving different from $4 d$ and $5 d$ ones.

Further, for each TM we considered the released energy during adsorption, $E_{a d s}$, to be equally distributed in all the degrees of freedom of the studied system, $3 \cdot \mathrm{N}$, being $\mathrm{N}$ the number of atoms contained in the cell $-48 \mathrm{C}$ atoms and $1 \mathrm{TM}$. By considering this and the application of the energy equipartition theorem, we estimated that those TMs which would retain in each degree of freedom an energy superior to $E_{\text {diff }}$ would then spontaneously trespass $\gamma$-graphyne in normal conditions. These TMs are those with a $\mathrm{Q}$ threshold below $0.7 \%(\mathrm{Pd}$ and $\mathrm{Au})$, although the amount of retained energy on the other above-commented cases $(\mathrm{Cr}$, $\mathrm{Mn}, \mathrm{Fe}, \mathrm{Rh}, \mathrm{Ag}, \mathrm{Ir}$, and $\mathrm{Pt}$ ) would help in their penetration as well in normal conditions, although one has to keep in mind that most of such TMs feature large $E_{a d s}$, which would 
imply that desorption could well be the limiting step of the sieving process, yet the reduced $E_{\text {ads }}$ of $\mathrm{Ag}$, and $\mathrm{Au}, 0.95$ and $1.02 \mathrm{eV}$, respectively, highlight such as the most feasible ones in this respect. In this respect, different aspects, such as the just commented remaining energy from the adsorption process, possible solvation effects, working temperatures, and the species chemical potential at the two sides of the g-graphyne membrane, defined by their activities concentrations in ideal conditions - constituting a thermodynamic driving force of the atomic trespassing, would contribute at making the desorption process after diffusion feasible.

Regardless of the previous, the obtained small diffusion barriers of late TMs are comparable to the easy permeability of $\mathrm{H}_{2}, \mathrm{He}$, and $\mathrm{H}_{2} \mathrm{O}$ on graphdiyne and graphtriyne $[23,24,32]$. In any case, note that the present permeation of TM adatoms is likely to be fostered when having larger acetylenic rings, starting from graphdiyne, but also considering other connectivities such as $\alpha-, \beta$-, and $\delta$-graphyne [6,32,51]. Notice how the $d$ series trends are similar to $E_{\text {diff, }}$ and that the TMs which occupy $\mathbf{H}_{\mathbf{3}}$ feature high Q values as well. Here the $d^{10} \mathrm{TMs}$ are out of the representation, as the $E_{d i f f}$ are larger than $E_{a d s}$, and so, imply that such atoms would rather detach from $\gamma$-graphyne when adsorb than diffusing across it. In that sense, $\gamma$-graphyne would be impermeable to them.

Another interesting aspect is whether such diffusion through the $\gamma$-graphyne sheet is competitive with respect the TM diffusion over it. To this end, the diffusion energies over the $\gamma$-graphyne, calculated likewise the present work in an earlier study [34], have been compared to the presently estimated trespassing energy barriers, listed in Table 1. For a better readability, the quotient in between the trespassing energy barrier with respect the diffusion over the $\gamma$-graphyne sheet, $\mathrm{Z}$, is plotted along the $d$ series in Fig. 4. From it several conclusions can be withdrawn, with similarities towards the Q plot in Fig. 3. On one hand, there is a general trend so the $E_{\text {diff }}$ get smaller when going along a $d$ series, and actually, the $E_{\text {diff }}$ values are generally smaller than the diffusion over the $\gamma$-graphyne layer for most cases. 
Only for group III and group XII TMs the ratio is well above one, whereas for group IV (Ti, $\mathrm{Zr}$, Hf), as well as early $4 d$ and $5 d \mathrm{TMs}(\mathrm{Nb}, \mathrm{Ta}, \mathrm{Mo}, \mathrm{W}$, and Re) and $3 d$ late TMs (Co, Ni, and $\mathrm{Cu}$ ), the values are close to one, implying that both processes, these are, the diffusion along or through the $\gamma$-graphyne layer, are competitive. For the rest, especially for late TMs of $4 d$ and $5 d$ series, the preference is clearly through the $\gamma$-graphyne sheet, ostensibly small for Fe, Pt, Pd, and Au.

Beyond the previous analysis, still an open question is whether the diffusion of TMs across $\gamma$-graphyne would be accompanied by water molecules, in particular since here, at variance with previous studies, we contemplate the deformation of the g-graphyne acetylenic ring so as to better allow the $\mathrm{H}_{2} \mathrm{O}$ permeation through. Previous MP2 studies on $\gamma$-graphyne revealed a very high $E_{\text {diff }}$ of $\sim 8 \mathrm{eV}$, although considering the graphyne model as a rigid body [32]. Furthermore, the possible stabilization of the TS by a hydrogen bond in between the trespassing $\mathrm{H}_{2} \mathrm{O}$ and one located on the other side of the $\gamma$-graphyne structure, known to stabilize the TS on graphdiyne, and therefore, reducing the $E_{\text {diff }}$ to merely $0.09 \mathrm{eV}$, seems to have been disregarded when considering $\gamma$-graphyne [32]. Here the step-wise TS location of the $\mathrm{H}_{2} \mathrm{O}$ molecule diffusing across the $\gamma$-graphyne layer has been considered, as commented, permitting the out-of-plane deformation of the $\gamma$-graphyne network, by anchoring the spatial position of the most distant graphyne $\mathrm{C}$ atom.

The diffusion profile is shown in Fig. 5. The $\mathrm{H}_{2} \mathrm{O}$ adsorption on $\mathbf{H}_{\mathbf{1}}$ position of $\gamma$ graphyne reveals an adsorption energy of solely $-0.08 \mathrm{eV}$, revealing the quite hydrophobic nature of $\gamma$-graphyne layers, which remains essentially planar during the interaction with the water molecule. The diffusion of $\mathrm{H}_{2} \mathrm{O}$ molecule across $\gamma$-graphyne through $\mathbf{H}_{3}$ implies a TS in which $\mathrm{H}_{2} \mathrm{O}$ contacts $\gamma$-graphyne through its $\mathrm{O}$ atom. The $\gamma$-graphyne structure is found to be dented, thus allowing for a better expansion of the acetylenic hole, ultimately making room 
for the $\mathrm{H}_{2} \mathrm{O}$ penetration across the $\gamma$-graphyne. This mechanism allows reducing the $E_{\text {diff }}$ barrier by almost $3 \mathrm{eV}$ compared to the aforementioned rigid block $E_{\text {diff }}$ estimation of $\sim 8 \mathrm{eV}$. However, the resulting $E_{\text {diff }}$ of $5.13 \mathrm{eV}$ is still excessively high so as not to allow the water permeation under normal conditions. All the attempts at stabilizing the TS through a $\mathrm{H}$ bond with another $\mathrm{H}_{2} \mathrm{O}$ molecule on the other side of the $\gamma$-graphyne structure were unsuccessful, revealing their independent adsorption at both sides of the $\gamma$-graphyne structure. Apparently, the graphyne acetylenic hole is too small, and the inherent $\gamma$-graphyne electron density screens the interaction in between $\mathrm{H}_{2} \mathrm{O}$ molecules located at opposites sides of the graphyne layer. From the comparison with previous studies, it seems clear that the pore size from graphdiyne size onwards is large enough to allow for the $\mathrm{H}$ bond stabilization mechanism [32]. However, for $\gamma$-graphyne, the present results imply that, when willing to separate TM cations in solution, water has to be present on both sides of the membrane if one wants to grant their solvation on both sides of the membrane.

In summary, the present DFT PBE simulations predict the free pass across $\gamma$-graphyne layers of $\mathrm{Fe}, \mathrm{Pd}, \mathrm{Pt}$, and $\mathrm{Au}$ atoms, all displaying penetration energy barriers below $0.05 \mathrm{eV}$. Furthermore, other $3 d(\mathrm{Cr}, \mathrm{Mn})$, Pt-group metals (Rh, Ir), and also Ag atoms feature $E_{\text {diff }}$ barriers below $0.5 \mathrm{eV}$, pointing for their possible permeability at affordable working temperature conditions. When considering TM desorption after sieving, Ag and Au are best candidates, combining both low $E_{\text {diff }}$ with low $E_{a d s}$ values. This graphyne is found to be impermeable to water, a point of possible technological interest, thus fostering the permeation of the just mentioned Pt-group and coinage metals, specially when driven by their different chemical potential at both sides of a $\gamma$-graphyne membrane. Thus, $\gamma$-graphyne is envisaged as a possible nanomaterial, which could be able to sieve such scarce and expensive metals from other elements present in a given water-based mixed solution. 


\section{Conclusions}

In this work, light is shed on the possible diffusion of TM atoms across a $\gamma$-graphyne layer, evaluating it as a possible membrane with sieving capabilities. The DFT PBE simulations on suited $\gamma$-graphyne models reveal that the diffusion of TM atoms across $\gamma$-graphyne is found to occur through the larger acetylenic holes instead of through the smaller graphenic ones. The located adsorption minima and diffusion TSs reveal quite different situations going along the periodic table $3 d, 4 d$, and $5 d$ series, with diffusion barriers ranging from almost negligible, as happens of $\mathrm{Au}$, displaying a diffusion energy barrier, $E_{\text {diff, }}$ of only $0.01 \mathrm{eV}$, to quite large, as happens for $\mathrm{Hg}$, with an $E_{\text {diff }}$ of $3.8 \mathrm{eV}$. Some $3 d$, Pt-group, and coinage metals (Fe, $\mathrm{Pd}, \mathrm{Pt}$, $\mathrm{Au}$ ) show $E_{\text {diff }}$ values below $0.05 \mathrm{eV}$, pointing for a free trespassing, while others $(\mathrm{Cr}, \mathrm{Mn}$, Ir, $\mathrm{Rh}, \mathrm{Ag}$ ) display $E_{\text {diff }}$ values below $0.5 \mathrm{eV}$, suggesting their possible trespassing at affordable conditions of temperature and pressure. The equal distribution of the adsorption energy of a TM atom in the systems degrees of freedom reveal that for some TMs ( $\mathrm{Pd}$ and $\mathrm{Au})$, the diffusion across the $\gamma$-graphyne layer would be thermodynamically and spontaneously feasible versus the anchoring itself process of the atoms to the $\gamma$-graphyne network. Last but not least, such TMs trespassing the $\gamma$-graphyne layer in a hypothetical aqueous solution is not accompanied by $\mathrm{H}_{2} \mathrm{O}$, as the $E_{\text {diff }}$ barrier is estimated to be $5.13 \mathrm{eV}$, even when accounting for possible $\gamma$-graphyne dent deformations to facilitate the molecular trespassing. All in all, $\gamma$ graphyne is pointed as a possible effective membrane to sieve late TM atoms, specially, $\mathrm{Pd}$ and $\mathrm{Au}$.

\section{Acknowledgements}

The work at SKKU was supported by the NRF (No. 2016R1A2B4012337) of Korea government (Ministry of Science, ICT \& Future Planning). The work at UB by Spanish 
Ministerio de Economía y Competitividad grants (CTQ2014-53987-R, CTQ2012-30751, and CTQ2015-64618-R) and Generalitat de Catalunya grants (2014SGR1582, 2014SGR97, 2017SGR13, and XRQTC). P.G. thanks Generalitat de Catalunya for his Serra Húnter Associate Professorship. F.V. thanks the Spanish Ministerio de Economía y Competitividad for the Ramón y Cajal (RyC) postdoctoral grant (RYC-2012-10129). Financial support from Spanish MINECO through the Excellence María de Maeztu program (grant MDM-20170767) is also fully acknowledged. 
Fig. 1. Schematic representation of the $\mathbf{H}_{1}$ and $\mathbf{H}_{3}$ sites of $\mathrm{TM}$ atoms on/in $\gamma$-graphyne labelled yellow spheres - from a top view (top image), and also the in-plane situation (bottom image). Carbon atoms correspond to green spheres.

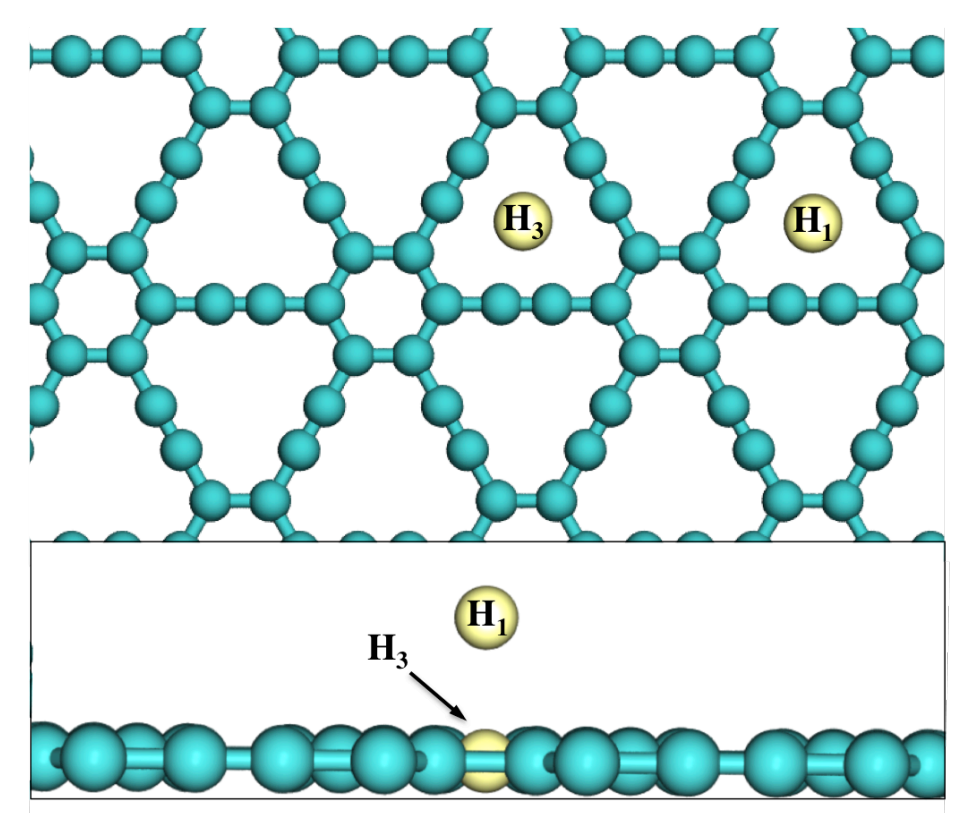


Fig. 2. Energy barriers for the diffusion of $3 d, 4 d$, and $5 d$ TMs across $\gamma$-graphyne layer $\left(E_{d i f f}\right)$, through the $\mathbf{H}_{3}$ site, as obtained by PBE DFT calculations. All values given in $\mathrm{eV}$.

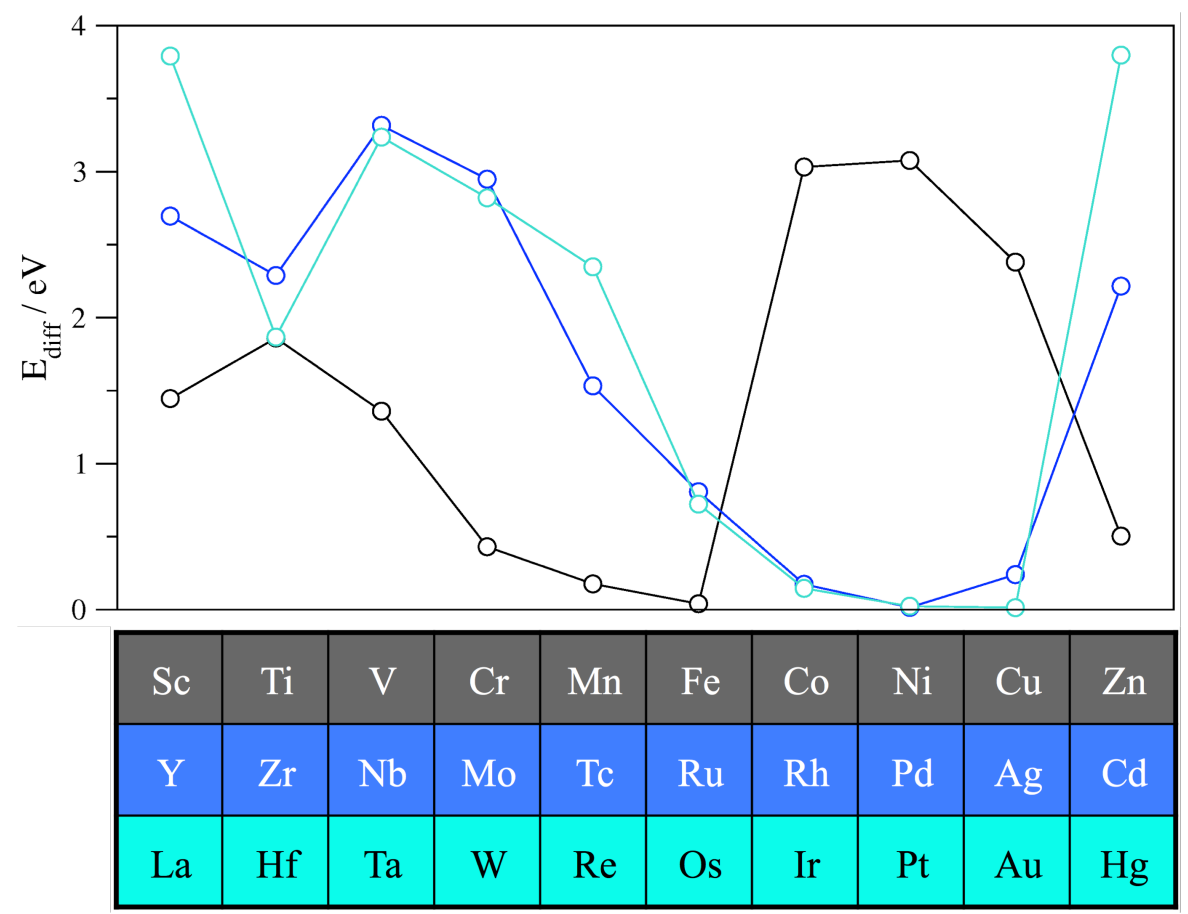


Fig. 3. Percentage (Q, in \%) of the diffusion energy barriers $\left(E_{\text {diff }}\right)$ across the $\gamma$-graphyne layer with respect to their adsorption energies $\left(E_{a d s}\right)$.

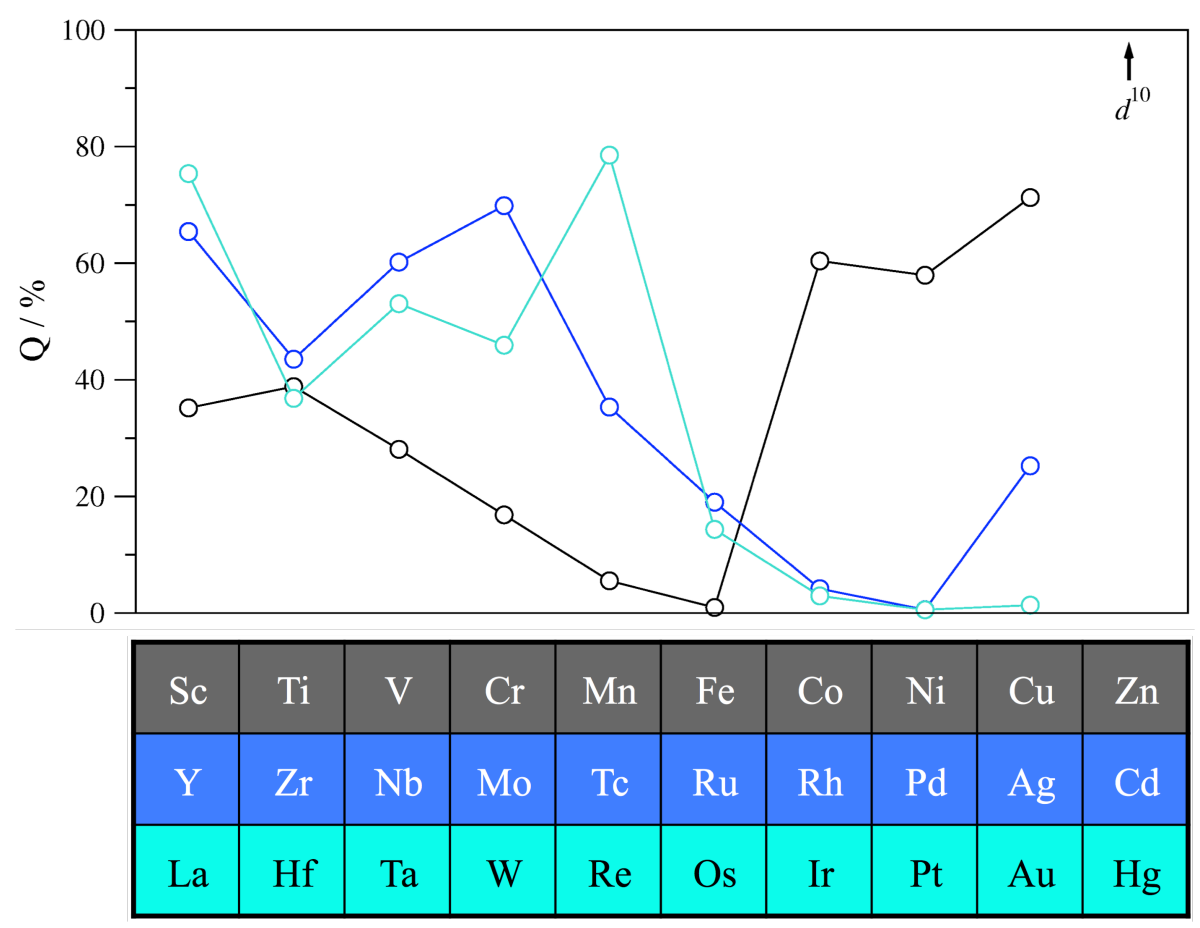


Fig. 4. Ratio, $Z$, in between the diffusion energy barriers $\left(E_{\text {diff }}\right)$ across the $\gamma$-graphyne layer and those above the $\gamma$-graphyne layer, reported elsewhere [34].

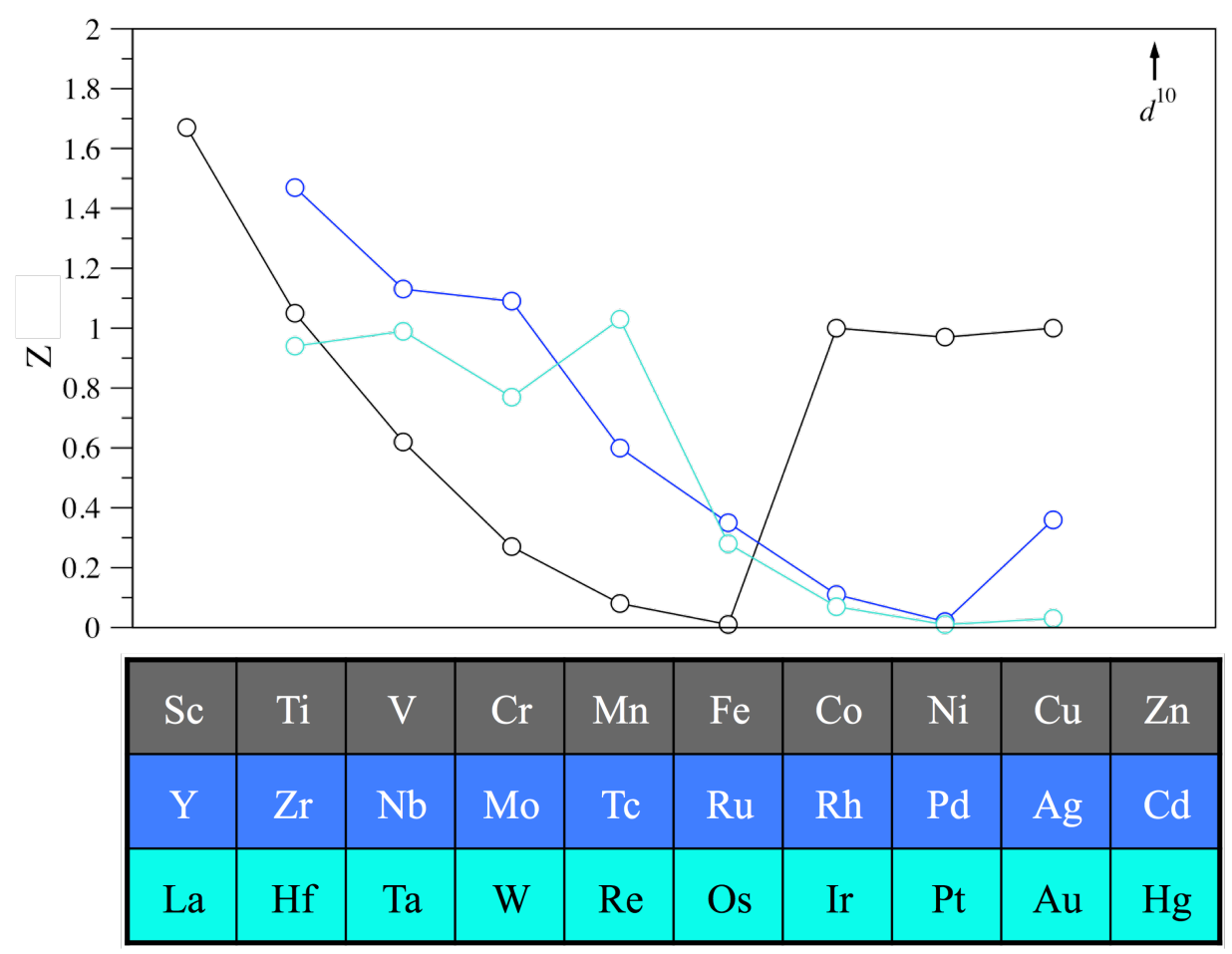


Fig. 5. $\mathrm{H}_{2} \mathrm{O}$ diffusion energy profile across the $\gamma$-graphyne structure. The insets show sided (bottom) and top (top) views on both minima and the found TS. C, O, and $\mathrm{H}$ atoms are shown as green, red, and white spheres, respectively.

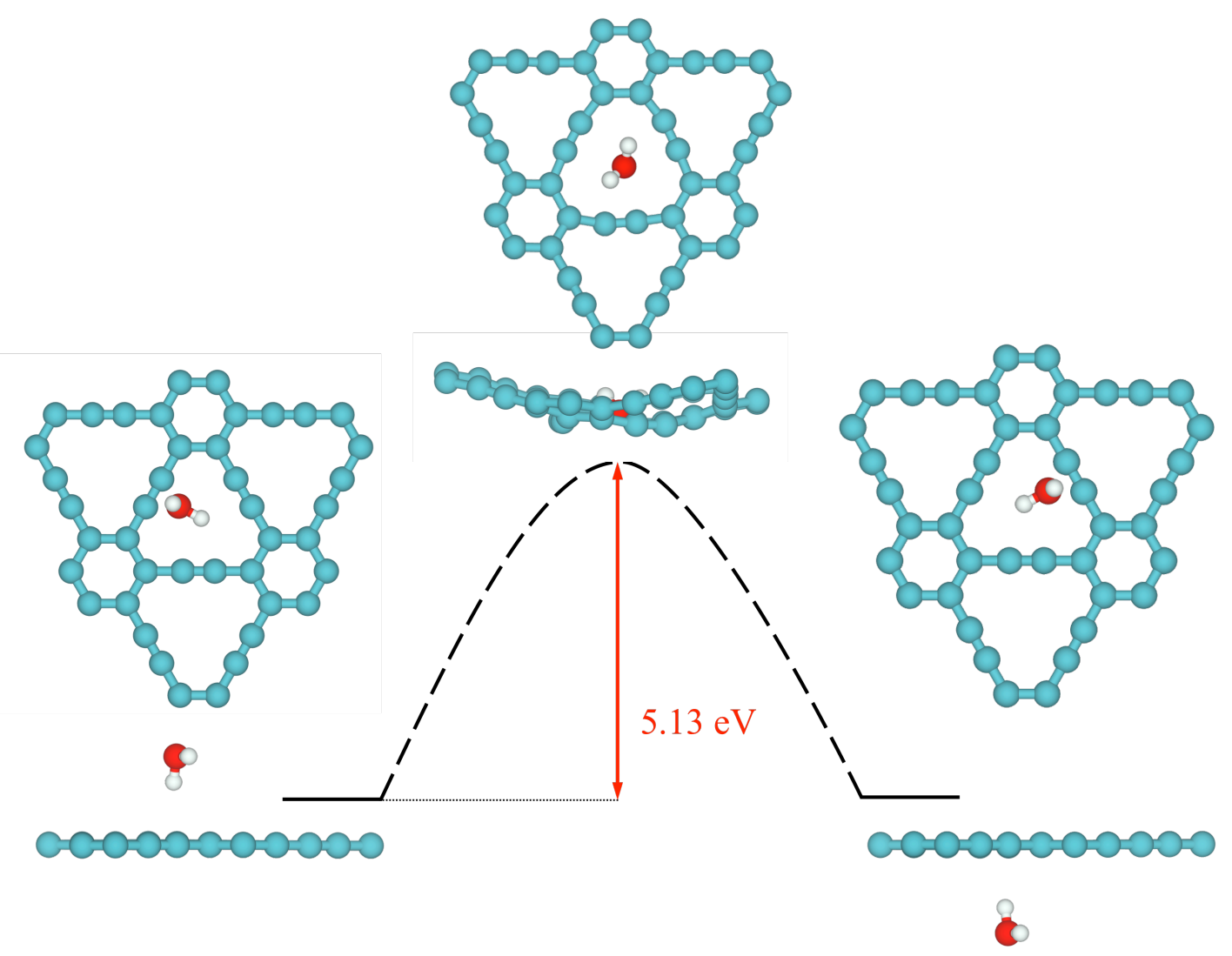


Table 1. Summary of $E_{\text {diff }}$ PBE results for $3 d, 4 d$, and $5 d$ TM across $\gamma$-graphyne, all values given in $\mathrm{eV}$ as well as computed $\mathrm{Q}$ values, in \%. The adsorption energies, $E_{\text {ads }}$, are shown for comparison as taken from literature [34].

\begin{tabular}{cccccccccccc}
\hline $\mathbf{3 d}$ & $\begin{array}{c}\boldsymbol{E}_{\text {diff }} \\
/ \mathbf{e V}\end{array}$ & $\begin{array}{c}\boldsymbol{E}_{\text {ads }} \\
/ \mathbf{e V}\end{array}$ & $\begin{array}{c}\boldsymbol{Q} \\
\boldsymbol{/ \%}\end{array}$ & $\mathbf{4 d}$ & $\begin{array}{c}\boldsymbol{E}_{\text {diff }} \\
/ \mathbf{e V}\end{array}$ & $\begin{array}{c}\boldsymbol{E}_{\text {ads }} \\
/ \mathbf{e V}\end{array}$ & $\begin{array}{c}\boldsymbol{Q} \\
/ \%\end{array}$ & $\mathbf{5 d}$ & $\begin{array}{c}\boldsymbol{E}_{\text {diff }} \\
/ \mathbf{e V}\end{array}$ & $\begin{array}{c}\boldsymbol{E}_{\text {ads }} \\
/ \mathbf{e V}\end{array}$ & $\begin{array}{c}\boldsymbol{Q} \\
\boldsymbol{/ \%}\end{array}$ \\
\hline \hline $\mathbf{S c}$ & 1.45 & 4.11 & 35 & $\mathbf{Y}$ & 2.70 & 4.12 & 65 & $\mathbf{L a}$ & 3.79 & 5.03 & 75 \\
$\mathbf{T i}$ & 1.86 & 4.79 & 39 & $\mathbf{Z r}$ & 2.29 & 5.26 & 44 & $\mathbf{H f}$ & 1.87 & 5.07 & 37 \\
$\mathbf{V}$ & 1.36 & 4.84 & 28 & $\mathbf{N b}$ & 3.32 & 5.51 & 60 & $\mathbf{T a}$ & 3.24 & 6.10 & 53 \\
$\mathbf{C r}$ & 0.43 & 2.56 & 17 & $\mathbf{M o}$ & 2.95 & 4.22 & 70 & $\mathbf{W}$ & 2.82 & 6.14 & 46 \\
$\mathbf{M n}$ & 0.18 & 3.20 & 6 & $\mathbf{T c}$ & 1.53 & 4.34 & 35 & $\mathbf{R e}$ & 2.35 & 2.99 & 79 \\
$\mathbf{F e}$ & 0.04 & 4.23 & 1 & $\mathbf{R u}$ & 0.81 & 4.25 & 19 & $\mathbf{O s}$ & 0.72 & 5.04 & 14 \\
$\mathbf{C o}$ & 3.03 & 5.02 & 60 & $\mathbf{R h}$ & 0.17 & 4.15 & 4 & $\mathbf{I r}$ & 0.15 & 4.96 & 3 \\
$\mathbf{N i}$ & 3.08 & 5.31 & 58 & $\mathbf{P d}$ & 0.02 & 2.70 & 1 & $\mathbf{P t}$ & 0.02 & 4.23 & 1 \\
$\mathbf{C u}$ & 2.38 & 3.34 & 71 & $\mathbf{A g}$ & 0.24 & 0.95 & 25 & $\mathbf{A u}$ & 0.01 & 1.02 & 1 \\
$\mathbf{Z n}$ & 0.50 & 0.02 & $-a$ & $\mathbf{C d}$ & 2.22 & 0.03 & $-a$ & $\mathbf{H g}$ & 3.80 & 0.02 & $-a$ \\
\hline
\end{tabular}

${ }^{a}$ Values above 1000 


\section{References}

[1] Y. Li, L. Xu, H. Liu, Y. Li, Graphdiyne and graphyne: from theoretical predictions to practical construction, Chem. Soc. Rev. 43 (2014) 2572-2586.

[2] V. Georgakilas, J.A. Perman, J. Tucek, R. Zboril, Broad family of carbon nanoallotropes: classification, chemistry, and applications of fullerenes, carbon dots, nanotubes, graphene, nanodiamonds, and combined superstructures, Chem. Rev. 115 (2015) 4744-4822.

[3] A.L. Ivanovskii, Graphynes and graphdyines, Prog. Solid State Chem. 41 (2013) 1-19.

[4] A.T. Balaban, C.C. Rentia, E. Ciupitu, Estimation of relative stability of several planar and tridimensional lattices for elementary carbon, Rev. Roum. Chim. 13 (1968) 231247.

[5] R.H. Baughman, H. Eckhardt, M. Kersetz, Structure-property predictions for new planar forms of carbon: layered phases containing $s p^{2}$ and $s p$ atoms, J. Chem. Phys. 87 (1987) 6687.

[6] D. Malko, C. Neiss, F. Viñes, A. Görling, Competition for graphene: graphynes with direction-dependent Dirac cones, Phys. Rev. Lett. 108 (2012) 086804.

[7] J. Chen, J. Xi, D. Wang, Z. Shuai, Carrier mobility in graphyne should be even larger than that in graphene: a theoretical prediction, J. Phys. Chem. Lett. 4 (2013) 14431448 .

[8] X. Qian, Z. Ning, Y. Li, H. Liu, C. Ouyang, Q. Chen, Y. Li, Construction of graphdiyne nanowires with high-conductivity and mobility, Dalton Trans. 41 (2012) 730-733.

[9] S. Wang, L. Yi, J.E. Halpert, X. Lai, Y. Liu, H. Cao, R. Yu, D. Wang, A novel and highly efficient photocatalyst based on P25-graphdiyne nanocomposite, Small 8 (2012) $265-271$.

[10] J.Y. Zhou, X. Gao, R. Liu, Z.Q. Xie, J. Yang, S.Q. Zhang, G. Zhang, H. Liu, Y. Li, J. Zhang, Z. Liu, Synthesis of graphdiyne nanowalls using acetylenic coupling reaction, J. Amer. Chem. Soc. 137 (2015) 7596-7599.

[11] Z. Li, M. Smeu, A. Rives, V. Maraval, R. Chauvin, M.A. Ratner, E. Borguet, Towards graphyne molecular electronics, Nat. Commun. 6 (2015) 6321.

[12] N. Yang, Y. Liu, H. Wen, Z. Tang, H. Zhao, Y. Li, D. Wang, Photocatalytic properties of graphdiyne and graphene modified $\mathrm{TiO}_{2}$ : from theory to experiment, ACS Nano 7 (2013) 1504-1512. 
[13] H.J. Hwang, J. Koo, M. Park, N. Park, Y. Kwon, H. Lee, Multilayer graphynes for Lithium ion battery anode, J. Phys. Chem. C 117 (2013) 6919-6923.

[14] S. Kim, P. Gamallo, F. Viñes, J.Y. Lee, F. Illas, Substrate-mediated single-atom isolation: dispersion of $\mathrm{Ni}$ and La on $\gamma$-graphyne, Theor. Chem. Acc. 136 (2017) 80.

[15] P. Wu, P. Du, H. Zhang, C.X. Cai, Graphyne as a promising metal-free electrocatalyst for oxygen reduction reactions in acidic fuel cells: a DFT study, J. Phys. Chem. C 116 ( 2012) 20472-20479.

[16] H.J. Hwang, Y. Kwon, H. Lee, Thermodynamically stable calcium-decorated graphyne as a hydrogen storage medium, J. Phys. Chem. C 116 (2012) 20220-20224.

[17] S.-H. Lee, S.-H. Jhi, A first-principles study of alkali-metal-decorated graphyne as oxygen-tolerant hydrogen storage media, Carbon 81 (2015) 418-425.

[18] D. Cohen-Tanugi, L.-C. Lin, J.C. Grossman, Multilayer nanoporous graphene membranes for water desalination, Nano Lett. 16 (2016) 1027-1033.

[19] J. Liu, G. Shi, H. Fang, Water flow in carbon-based nanoporous membranes impacted by interactions between hydrated ions and aromatic rings, Nanotechnology 28 (2017) 084004

[20] Z. Song, Z. Xu, Ultimate osmosis engineered by the pore geometry and functionalization of carbon nanostructures, Sci. Rep. 5 (2015) 10597.

[21] A. Nicolaï, B.G. Sumpter, V. Meunier, Tunable water desalination across graphene oxide framework membranes. Phys. Chem. Chem. Phys. 16 (2014) 8646-8654.

[22] S. Dervin, D.D. Dionysiou, S.C. Pillai, 2D nanostructures for water purification: graphene and beyond, Nanoscale 8 (2016) 15115-15131.

[23] S.W. Cranford, M.J. Buehler, Selective hydrogen purification through graphdiyne under ambient temperature and pressure, Nanoscale 4 (2012) 4587.

[24] M. Bartolomei, E. Carmona-Novillo, M.I. Hernández, J. Campos-Martínez, F. Pirani, G. Giorgi, Graphdiyne pores: "ad hoc" openings for helium separation applications. J. Phys. Chem. C 118 (2014) 29966-29972.

[25] M.I. Hernández, M. Bartolomei, J. Campos-Martínez, Transmission of helium isotopes through graphdiyne pores: tunneling versus zero point energy effects, J. Phys. Chem. A 119 (2015) 10743-10749.

[26] X. Zhang, J.-G. Gai, Single-layer graphyne membranes for superexcellent brine separation in forward osmosis, RSC Adv. 5 (2015) 68109-68116. 
[27] C. Zhu, H. Li, X.C. Zeng, E.G. Wang, S. Meng, Quantized water transport: ideal desalination through graphyne-4 membrane, Sci. Rep. 3 (2013) 3163.

[28] J.L. Kou, X.Y. Zhou, H.J. Lu, F.M. Wu, J.T. Fan, Graphyne as the membrane for water desalination, Nanoscale 6 (2014) 1865-1870.

[29] S. Lin, M.J. Buehler, Mechanics and molecular filtration performance of graphyne nanoweb membranes for selective water purification, Nanoscale 5 (2013) 11801-11807.

[30] M. Xue, H. Qiu, W. Guo, Exceptionally fast water desalination at complete salt rejection by pristine graphyne monolayers, Nanotechnology 24 (2013) 505720.

[31] L.-P. Wu, X. Zhang, Y. Chen, L.-Y. Chen, J.-G. Gai, Fast water transmission of zigzag graphyne-3 nanotubes, RSC Adv. 6 (2016) 109099-109104.

[32] M. Bartolomei, E. Carmona-Novillo, M.I. Hernández, J. Campos-Martínez, F. Pirani, G. Giorgi, K. Yamashita, Penetration barrier of water through graphynes' pores: firstprinciples predictions and force field optimization, J. Phys. Chem. Lett. 5 (2014) 751755.

[33] S. Bagheri, A. Shameli, G. Fakhrpour, Molecular investigation of water adsorption on graphene and graphyne surfaces, Physica E 90 (2017) 123-130.

[34] S. Kim, A. Ruiz Puigdollers, P. Gamallo, F. Viñes, J.Y. Lee, functionalization of $\gamma$ graphyne by transition metal adatoms, Carbon 120 (2017) 63-70.

[35] M. Manadé, F. Viñes, F. Illas, Transition metal adatoms on graphene: a systematic density functional study, Carbon 95 (2015) 525-534.

[36] B. S. Lim, A. Rahtu, R. G. Gordon, Atomic layer deposition of transition metals, Nat. Mater. 2 (2003) 749-754.

[37] S. Vajda, M. J. Pellin, J. P. Greeley, C. L. Marshall, L. A. Curtiss, G. A. Ballentine, J. W. Elam, S. Catillon-Mucherie, P. C. Redfern, F. Mehmood, P. Zapol, R. G. Gordon, Subnanometre platinum clusters as highly active and selective catalysts for the oxidative dehydrogenation of propane, Nat. Mater. 8 (2009) 213-216.

[38] N. F. Dalleska, K. Honma, L. S. Sunderlin, P. B. Armentrout, Solvation of transition metal ions by water. Sequential binding energies of $\mathrm{M}^{+}\left(\mathrm{H}_{2} \mathrm{O}\right)_{x}(x=1-4)$ for $\mathrm{M}=\mathrm{Ti}$ to $\mathrm{Cu}$ determined by collision-induced dissociation, J. Am. Chem. Soc. 116 (1994) 35193528.

[39] B. Bandyopadhyay, K. N. Reishus, M. A. Duncan, Infrared spectroscopy of solvation in small $\mathrm{Zn}^{+}\left(\mathrm{H}_{2} \mathrm{O}\right)_{\mathrm{n}}$ complexes, J. Phys. Chem. A 117 (2013) 7794-7803. 
[40] G. Kresse, J. Furthmüller, Efficient iterative schemes for $a b$ initio total-energy calculations using a plane-wave basis set, Phys. Rev. B 54 (1996) 11169-11186.

[41] P.E. Blöchl, Projector augmented-wave method, Phys. Rev. B 50 (1994) 17953-17979.

[42] J.P. Perdew, K. Burke, M. Ernzerhof, Generalized gradient approximation made simple, Phys. Rev. Lett. 77 (1996) 3865-3868.

[43] B. Kang, H. Liu, J.Y. Lee, Oxygen adsorption on single layer graphyne: a DFT study. Phys. Chem. Chem. Phys. 16 (2014) 974-980.

[44] A. Ruiz Puigdollers, G. Alonso, P. Gamallo, First-principles study of structural, elastic and electronic properties of $\alpha$-, $\beta$ - and $\gamma$-graphyne, Carbon 96 (2016) 879-887.

[45] P. Janthon, S.M. Kozlov, F. Viñes, J. Limtrakul, F. Illas, Establishing the accuracy of broadly used density functionals in describing bulk properties of transition metals. J. Chem. Theory Comput. 9 (2013) 1631-1640.

[46] P. Janthon, S.J. Luo, S.M. Kozlov, F. Viñes, J. Limtrakul, D.G. Truhlar, F. Illas, Bulk properties of transition metals: a challenge for the design of universal density functionals, J. Chem. Theory Comput. 10 (2014) 3832-3839.

[47] L. Vega, J. Ruvireta, F. Viñes, F. Illas, Jacob's ladder as sketched by escher: assessing the performance of broadly used density functionals on transition metal surface properties, J. Chem. Theory Comput. 14 (2018) 395-403.

[48] H.J. Monkhorst, J.D. Pack, Special points for Brillouin-zone integrations, Phys. Rev. B 13 (1976) 5188-5192.

[49] S. Grimme, Semiempirical GGA-type density functional constructed with a long-range dispersion correction, J. Comput. Chem. 27 (2006) 1787-1799.

[50] A.U. Nilekar, J. Greeley, M. Mavrikakis, A simple rule of thumb for diffusion on transition-metal surfaces, Angew. Chem. Int. Ed. 45 (2006) 7046-7049.

[51] H. Zhou, S. Lou, F. Li, Y. Qu, Carbon nanoribbons and nanotubes based on deltagraphyne: A first-principles study, Physica E 78 (2016) 19-24. 